\title{
Lambda-nucleon force from lattice QCD
}

\author{
Hidekatsu Nemura* \\ Strangeness Nuclear Physics Laboratory, Nishina Center for Accelerator-Based Science, \\ RIKEN,Wako, 351-0198, Japan \\ E-mail: nemura@riken.jp
}

\section{Noriyoshi Ishii}

Center for Computational Sciences, University of Tsukuba, Tsukuba 305-8571, Japan

E-mail: ishii@ribf.riken.jp

\section{Sinya Aoki}

Graduate School of Pure and Applied Sciences, University of Tsukuba, Tsukuba 305-8571, Japan

Riken BNL Research Center, Brookhaven National Laboratory, Upton, New York 11973, USA

E-mail: saoki@het.ph.tsukuba.ac.jp

\section{Tetsuo Hatsuda}

Department of Physics, University of Tokyo, Tokyo 113-0033, Japan

E-mail: hatsuda@phys.s.u-tokyo.ac.jp

\section{for PACS-CS Collaboration}

We study the $\Lambda$-nucleon $(\Lambda N)$ force by using lattice QCD. The Bethe-Salpeter amplitude is calculated for the lowest scattering state of the $\Lambda N$ so as to obtain the $\Lambda N$ potential. The numerical calculation is twofold: (i) Full lattice QCD by using $2+1$ flavor PACS-CS gauge configurations with, $\beta=1.9$, corresponding to the lattice spacing of $a=0.0907(13) \mathrm{fm}$, on a $32^{3} \times 64$ lattice. A set of parameter $\left(\kappa_{u d}, \kappa_{s}\right)=(0.13770,0.13640)$ is used, which corresponds to $m_{\pi} \approx 300 \mathrm{MeV}$ and $m_{K} \approx 594 \mathrm{MeV}$. The spatial lattice volume corresponds to $(2.86 \mathrm{fm})^{3}$. (ii) Quenched lattice QCD with $\beta=5.7$, the lattice spacing of $a=0.1416(9) \mathrm{fm}$, on the $32^{3} \times 48$ lattice. Two sets of hopping parameters $\left(\kappa_{u d}, \kappa_{s}\right)=(0.1665,0.1643),(0.1670,0.1643)$ are used. The spatial lattice volume is $(4.5 \mathrm{fm})^{3}$. For the full QCD, we find that the $\Lambda p$ has a relatively strong (weak) repulsive core in the ${ }^{1} S_{0}\left({ }^{3} S_{1}\right)$ channel at short distance, while the potential has slight attractive region at medium distance. The lowest scattering energy in the finite lattice volume is calculated; Slightly negative values obtained in both spin channels. For the quenched QCD, we find that the results are qualitatively in agreement with those in the full QCD calculation.

The XXVI International Symposium on Lattice Field Theory

July 14 - 19, 2008

Williamsburg, Virginia, USA

\footnotetext{
*Speaker.
} 


\section{Introduction}

Study of the hyperon-nucleon $(Y N)$ and hyperon-hyperon $(Y Y)$ interactions is one of the important tasks in the contemporary nuclear physics. These interactions are the bases to explore the strange nuclear systems, in which hyperons (or strange quarks) are embedded in normal nuclear systems as "impurities" [1]. Various issues, such as spin- and flavor-dependence, effect of the flavor $S U$ (3) breaking, effect of hyperonic mixing (e.g., $\Lambda N-\Sigma N$ and $\Lambda \Lambda-\Xi N$ ), are the topics to be solved experimentally and/or theoretically. For example, recent systematic study (e.g., Ref. [2]) for light (s-shell) $\Lambda$ hypernuclei $\left({ }_{\Lambda}^{3} \mathrm{H},{ }_{\Lambda}^{4} \mathrm{H},{ }_{\Lambda}^{4} \mathrm{He}\right.$ and ${ }_{\Lambda}^{5} \mathrm{He}$ ) suggests that the $\Lambda N$ interaction in the ${ }^{1} S_{0}$ channel is more attractive than that in the ${ }^{3} S_{1}$ channel. However, the present $Y N$ and the $Y Y$ interactions have large uncertainties, because the scattering experiments are either difficult or impossible due to the short life-time of hyperons. Theoretically, it should be desirable to understand the $Y N$ and $Y Y$ interaction (or, in more general, baryon-baryon interaction) based on the dynamics of quarks and gluons as fundamental degrees of freedom. If one can perform such an appropriate deduction along the theory of quantum chromodynamics (QCD), they should have a reliable prediction regarding the $Y N$ and $Y Y$ potentials.

Recently, the lattice QCD studies have been performed for not only the $N N$ potential but also the $\Xi N$ potential in quenched QCD [3, 4]. See also Refs. [5, 6, 7] for the recent developments. The lattice QCD calculations focusing on the scattering parameters for $\Lambda N$ system based on Lüscher's formula have been reported in Refs. [8, 9]. The purpose of this report is to calculate the $\Lambda N$ potentials by using the full and quenched QCD gauge configurations. The main results of this work are obtained by using the $2+1$ flavor PACS-CS gauge configurations with the spatial lattice volume $(2.86 \mathrm{fm})^{3}$ [10]. We also report the results calculated by using quenched lattice QCD with larger spatial lattice volume $(4.5 \mathrm{fm})^{3}$.

\section{Formulation}

The basic formulation is already given in Refs. [3, 4, 5]. (See also Refs. [11, 12].) In this report, we briefly explain the procedure to obtain the potential from lattice QCD together with some technical point newly introduced in the present work. We start from an effective Schrödinger equation for the equal-time Bethe-Salpeter (BS) wave function:

$$
-\frac{1}{2 \mu} \nabla^{2} \phi(\vec{r})+\int U\left(\vec{r}, \vec{r}^{\prime}\right) \phi\left(\vec{r}^{\prime}\right) d^{3} r^{\prime}=E \phi(\vec{r}) .
$$

Here $\mu=m_{\Lambda} m_{N} /\left(m_{\Lambda}+m_{N}\right)$ and $E \equiv k^{2} /(2 \mu)$ are the reduced mass of the $\Lambda N$ system and the non-relativistic energy in the center-of-mass frame, respectively. We consider the low-energy scattering state so that the nonlocal potential can be rewritten by derivative expansion [13], $U\left(\vec{r}, \vec{r}^{\prime}\right)=$ $V_{\Lambda N}(\vec{r}, \vec{\nabla}) \boldsymbol{\delta}\left(\vec{r}-\vec{r}^{\prime}\right)$. The general expression of the potential $V_{\Lambda N}$ is known to be [14]

$$
V_{\Lambda N}=V_{0}(r)+V_{\sigma}(r)\left(\vec{\sigma}_{\Lambda} \cdot \vec{\sigma}_{N}\right)+V_{T}(r) S_{12}+V_{L S}(r)\left(\vec{L} \cdot \vec{S}_{+}\right)+V_{A L S}(r)\left(\vec{L} \cdot \vec{S}_{-}\right)+O\left(\nabla^{2}\right) .
$$

Here $S_{12}=3\left(\vec{\sigma}_{\Lambda} \cdot \vec{n}\right)\left(\vec{\sigma}_{N} \cdot \vec{n}\right)-\vec{\sigma}_{\Lambda} \cdot \vec{\sigma}_{N}$ is the tensor operator with $\vec{n}=\vec{r} /|\vec{r}|, \vec{S}_{ \pm}=\left(\vec{\sigma}_{N} \pm \vec{\sigma}_{\Lambda}\right) / 2$ are symmetric ( + ) and antisymmetric (-) spin operators, $\vec{L}=-i \vec{r} \times \vec{\nabla}$ is the orbital angular momentum 
operator. We note that the antisymmetric spin-orbit forces $\left(V_{A L S}\right.$ and $\left.V_{A L S \tau}\right)$ do not arise in the $N N$ case because of the identical nature of the nucleon within the isospin symmetry.

First we calculate the four-point correlator by using the lattice QCD

$$
G_{\alpha \beta ; \alpha^{\prime} \beta^{\prime}}\left(\vec{r}, t-t_{0}\right)=\sum_{\vec{X}}\left\langle 0\left|p_{\alpha}(\vec{X}+\vec{r}, t) \Lambda_{\beta}(\vec{X}, t){\overline{\Lambda^{\prime}}}_{\beta^{\prime}}\left(t_{0}\right){\overline{p^{\prime}}}_{\alpha^{\prime}}\left(t_{0}\right)\right| 0\right\rangle,
$$

where the summation over $\vec{X}$ is to select the state with zero total momentum. The $p_{\alpha}(x)$ and $\Lambda_{\beta}(y)$ denote the interpolating fields for proton and $\Lambda$

$$
\begin{aligned}
& p_{\alpha}(x)=\varepsilon_{a b c}\left(u_{a}(x) C \gamma_{5} d_{b}(x)\right) u_{c \alpha}(x) \\
& \Lambda_{\beta}(y)=\varepsilon_{a b c}\left\{\left(d_{a}(y) C \gamma_{5} s_{b}(y)\right) u_{c \beta}(y)+\left(s_{a}(y) C \gamma_{5} u_{b}(y)\right) d_{c \beta}(y)-2\left(u_{a}(y) C \gamma_{5} d_{b}(y)\right) s_{c \beta}(y)\right\}
\end{aligned}
$$

and $\overline{\Lambda^{\prime}}{ }_{\beta^{\prime}}\left(t_{0}\right)$ and $\overline{p^{\prime}}{ }_{\alpha^{\prime}}\left(t_{0}\right)$ denote the wall source

$$
\begin{gathered}
p_{\alpha^{\prime}}^{\prime}(t)=\sum_{\vec{x}_{1}, \vec{x}_{2}, \vec{x}_{3}} \varepsilon_{a b c}\left(u_{a}\left(\vec{x}_{1}, t\right) C \gamma_{5} d_{b}\left(\vec{x}_{2}, t\right)\right) u_{c \alpha^{\prime}}\left(\vec{x}_{3}, t\right), \\
\Lambda_{\beta^{\prime}}^{\prime}(t)=\sum_{\vec{y}_{1}, \vec{y}_{2}, \vec{y}_{3}} \varepsilon_{a b c}\left\{\left(d_{a}\left(\vec{y}_{1}, t\right) C \gamma_{5} s_{b}\left(\vec{y}_{2}, t\right)\right) u_{c \beta^{\prime}}\left(\vec{y}_{3}, t\right)+\left(s_{a}\left(\vec{y}_{1}, t\right) C \gamma_{5} u_{b}\left(\vec{y}_{2}, t\right)\right) d_{c \beta^{\prime}}\left(\vec{y}_{3}, t\right)\right. \\
\left.\quad-2\left(u_{a}\left(\vec{y}_{1}, t\right) C \gamma_{5} d_{b}\left(\vec{y}_{2}, t\right)\right) s_{c \beta^{\prime}}\left(\vec{y}_{3}, t\right)\right\} .
\end{gathered}
$$

The Fast Fourier Transformation (FFT) algorithm is imposed into the actual $\mathrm{C}++$ code to reduce the computational time, which makes the calculation at all of the spatial points $\left(32^{3}\right)$ possible. (In the previous work for the $\Xi^{0} p$ system, we calculate only on the $x$-, $y$ - and $z$-axes and the nearest neighbors in the long distance region. [4])

In the Monte Carlo calculations, noise reductions are made for the four-point correlator (2.3) obtained from lattice QCD in order to restore (i) the rotational (cubic group) symmetry, (ii) the spatial reflection symmetry, (iii) the charge conjugation and time-reversal symmetry. Then, we perform the spin projection for the source to obtain the wave function with particular spin component $(J=0,1)$.

$$
F_{p_{\alpha} \Lambda_{\beta}}\left(\vec{r}, t-t_{0} ; J, M\right)=\sum_{\alpha^{\prime} \beta^{\prime}} P_{\alpha^{\prime} \beta^{\prime}}^{(J M)} G_{\alpha \beta ; \alpha^{\prime} \beta^{\prime}}\left(\vec{r}, t-t_{0}\right),
$$

See Ref. [7] for detail. Finally, the desirable wave function $\phi_{\alpha \beta}$ is obtained from the projected four-point correlator at large $t-t_{0}$ :

$$
\begin{aligned}
F_{p_{\alpha} \Lambda_{\beta}}\left(\vec{r}, t-t_{0} ; J, M\right) & =\sum_{n} A_{n} \sum_{\vec{X}}\left\langle 0\left|p_{\alpha}(\vec{X}+\vec{r}, t) \Lambda_{\beta}(\vec{X}, t)\right| E_{n}\right\rangle e^{-E_{n}\left(t-t_{0}\right)} \\
& \approx A_{0} \phi_{\alpha \beta}(\vec{r} ; J, M) e^{-E_{0}\left(t-t_{0}\right)} \quad\left(t-t_{0} \rightarrow \text { large }\right) .
\end{aligned}
$$

Here $E_{n}\left(\left|E_{n}\right\rangle\right)$ is the eigen-energy (eigen-state) of the six-quark system with the particular quantum number $\left(J^{\pi}, M\right)$, and $A_{n}=\sum_{\alpha^{\prime} \beta^{\prime}} P_{\alpha^{\prime} \beta^{\prime}}^{(J M)}\left\langle E_{n}\left|\overline{\Lambda^{\prime}} \beta_{\beta^{\prime}} \bar{p}^{\prime}{ }^{\prime}\right| 0\right\rangle$. For simplicity, we consider the effective central potential, $V_{C}(r ; J=0)=V_{0}(r)-3 V_{\sigma}(r)$, for ${ }^{1} S_{0}$ and, $V_{C}(r ; J=1)=V_{0}(r)+V_{\sigma}(r)+\cdots$, for ${ }^{3} S_{1}$, where $\cdots$ is the higher order contribution from noncentral force such as $V_{T}(r)$, which is expected to be small in the present calculation. The effective central potential $V_{C}(r ; J)$ is obtained by focusing only on the $S$-wave component of the wave function in each spin channel $J$ :

$$
V_{C}(r ; J)=E_{J}+\frac{1}{2 \mu} \frac{\vec{\nabla}^{2} \phi(r ; J)}{\phi(r ; J)} .
$$




\section{Numerical calculation}

\section{1 $N_{f}=2+1$ QCD}

Main results in this report are obtained by using the gauge configuration generated by PACSCS collaboration with $2+1$ flavor full QCD [10]; The Iwasaki gauge action and the nonperturbatively $O(a)$-improved Wilson quark action are employed. Calculations are carried out at $\beta=1.9$ on a $32^{3} \times 64$ lattice, corresponding to $a=0.0907(13)$ fm where $a$ is the lattice spacing at the physical point [10]. A set of hopping parameters $\left(\kappa_{u d}, \kappa_{s}\right)=(0.13770,0.13640)$ is used in this work. Several light hadron masses obtained by PACS-CS are shown in Table 1 . The BS wave function is obtained by employing the wall source at the time-slice $t_{0}=0$, and the Dirichlet boundary condition is imposed along the temporal direction on the time-slice $t=32$ with the Coulomb gauge fixing. The present result of the BS wave function is calculated with $N_{\text {conf }}=422$, where $N_{\text {conf }}$ is the number of gauge configurations.

\subsection{Quenched QCD with larger spatial volume}

In this case, we use the plaquette gauge action and the Wilson fermion action with the gauge coupling $\beta=5.7$ on the $32^{3} \times 48$ lattice. The periodic boundary condition is imposed for quarks in the spatial direction. The wall source is placed at $t_{0}=0$ with the Coulomb gauge fixing and the Dirichlet boundary condition is imposed at $t=24$ in the temporal direction. These setup are essentially the same as our previous calculations for the $N N$ and $\Xi N$ potentials [3, 4] except for the treatment of the temporal part. The lattice spacing at the physical point is determined as $a=0.1416(9) \mathrm{fm}(1 / a=1.393(9) \mathrm{GeV})$ from $m_{\rho}=770 \mathrm{MeV}$. The hopping parameter for the strange quark mass is given by $\kappa_{s}=0.16432(6)$ from $m_{K}=494 \mathrm{MeV}$. The spatial lattice volume is $(4.5 \mathrm{fm})^{3}$, which is enough to accommodate two baryons. The present result of the BS wave function in quenched QCD is obtained with $N_{\text {conf }}=550$.

\section{Results and Discussion}

\section{1 $N_{f}=2+1$ QCD}

The left panel of Figure 1 shows the wave function obtained at the time slice $t-t_{0}=7$. The red circle (blue triangle) corresponds to the ${ }^{1} S_{0}\left({ }^{3} S_{1}\right)$ channel. In order to find the ground state

\begin{tabular}{|c|c|c|c|c|c|c|c|c|}
\hline & $m_{\pi}$ & $m_{\rho}$ & $m_{K}$ & $m_{K^{*}}$ & $m_{N}$ & $m_{\Lambda}$ & $m_{\Sigma}$ & $m_{\Xi}$ \\
\hline \multicolumn{9}{|c|}{$2+1$ flavor QCD by PACS-CS with $\left(\kappa_{u d}, \kappa_{s}\right)=(0.13770,0.13640)$} \\
\hline Ref.[10] & 296(3) & $848(20)$ & $594(2)$ & $985(8)$ & 1093(19) & $1254(14)$ & $1315(15)$ & $1448(10)$ \\
\hline \multicolumn{9}{|c|}{ quenched QCD with $\beta=5.7, \kappa_{s}=\mathbf{0 . 1 6 4 3}$} \\
\hline$\kappa_{u d}=0.1665$ & $514(1)$ & $859(3)$ & $607(1)$ & $904(3)$ & $1287(8)$ & $1345(6)$ & $1368(6)$ & $1412(5)$ \\
\hline$\kappa_{u d}=0.1670$ & $465(1)$ & $840(6)$ & $588(1)$ & $894(3)$ & $1234(11)$ & 1308(7) & $1341(8)$ & 1396(5) \\
\hline Exp. & 135 & 770 & 494 & 892 & 940 & 1116 & 1190 & 1320 \\
\hline
\end{tabular}

Table 1: Hadron masses in the unit of MeV. The results for $2+1$ flavor QCD by PACS-CS is taken from Ref. [10]. $N_{\text {conf }}=560$ in quenched QCD. 

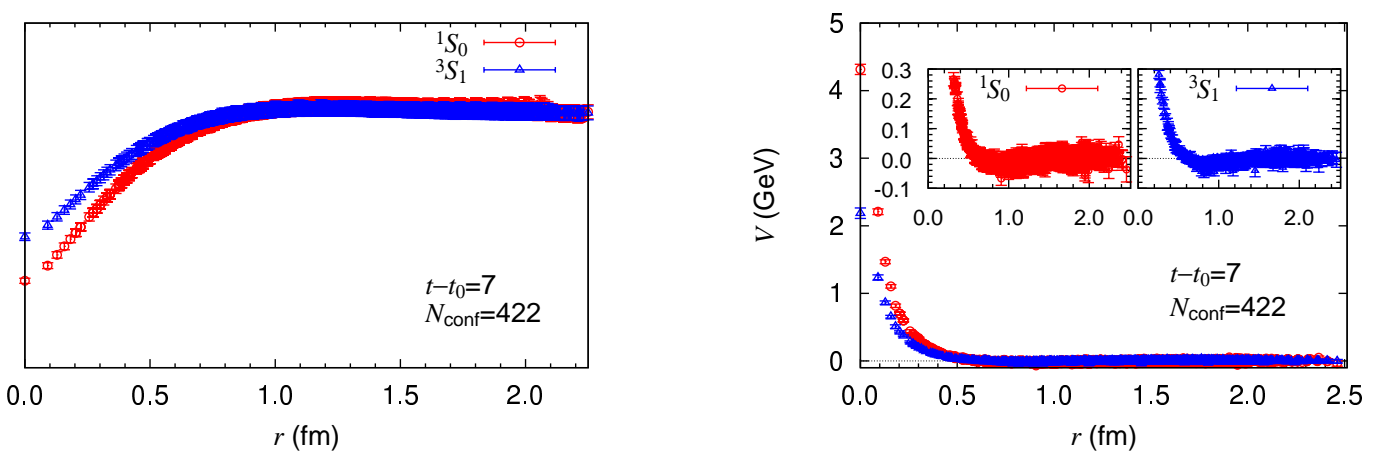

Figure 1: Left: The radial wave function of $\Lambda p$, in ${ }^{1} S_{0}$ (red circle) and ${ }^{3} S_{1}$ (blue triangle) channels, obtained by using the PACSCS gauge configurations at $t-t_{0}=7 . N_{\text {conf }}=422$ gauge configurations are used. Right: The effective central potential for $\Lambda p$, in the ${ }^{1} S_{0}$ (red circle) and ${ }^{3} S_{1}$ (blue triangle), obtained from the wave function at time slice $t-t_{0}=7$. The left (right) inset shows its enlargement in the ${ }^{1} S_{0}\left({ }^{3} S_{1}\right)$ channel.

saturation of the $\Lambda p$ system, we define the "effective mass" of the wave function:

$$
m_{\mathrm{eff}}\left(t-t_{0}, \vec{r}\right) \equiv \log \left(\frac{F_{p \Lambda}\left(\vec{r}, t-t_{0}\right)}{F_{p \Lambda}\left(\vec{r},(t+1)-t_{0}\right)}\right) .
$$

Figure 2 shows the effective mass at several spatial points. The plateaux starting appears at the time slice $t-t_{0}=6$ in the ${ }^{3} S_{1}$ channel (in the right panel in Fig. 2) while the plateaux starting seems to appear at the time slice around $t-t_{0}=7$ in the ${ }^{1} S_{0}$ channel (in the left panel). We need more statistics. In this report, we show the results obtained at the time slice $t-t_{0}=7$.

As is shown in Ref. [12], the non-relativistic energy $E=k^{2} /(2 \mu)$ is determined by fitting the wave function in the asymptotic region in terms of the Green's function

$$
G\left(\vec{r}, k^{2}\right)=\frac{1}{L^{3}} \sum_{\vec{p} \in \Gamma} \frac{1}{p^{2}-k^{2}} \mathrm{e}^{i \vec{p} \cdot \vec{r}}, \quad \Gamma=\left\{\vec{p} ; \vec{p}=\vec{n} \frac{2 \pi}{L}, \vec{n} \in Z^{3}\right\}
$$

which is the solution of $\left(\triangle+k^{2}\right) G\left(\vec{r}, k^{2}\right)=-\delta_{L}(\vec{r})$ with $\delta_{L}(\vec{r})$ being the periodic delta function [11, 12]. We attempted to make a fit in the asymptotic region $(15 \leq|\vec{r}| \leq 16)$ at the time slice $t-t_{0}=7$. The fitting region is chosen so that the potential becomes zero within the errorbars. The
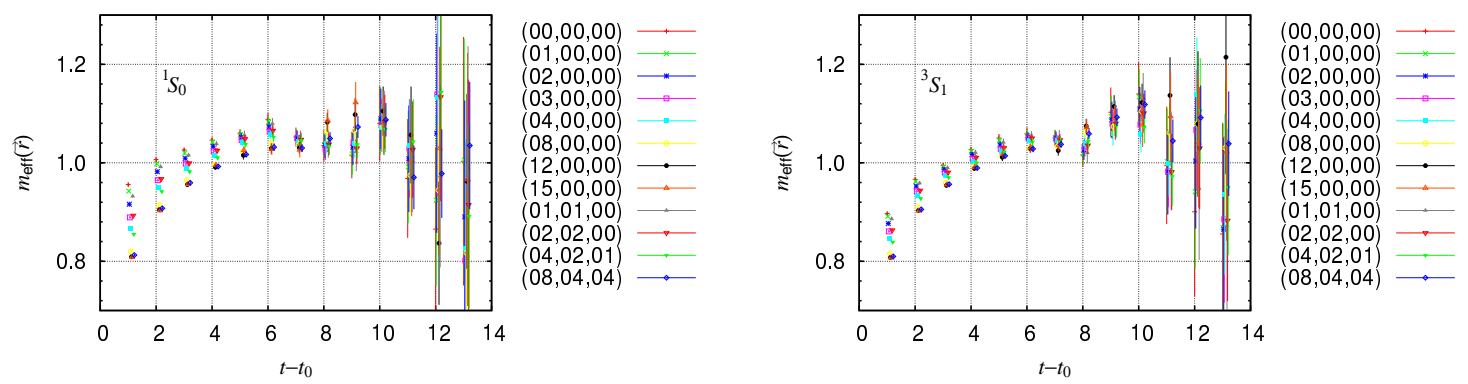

Figure 2: The effective mass of the wave function for $\Lambda p$ at several spatial points $(x, y, z)$, in the ${ }^{1} S_{0}$ (left) and the ${ }^{3} S_{1}$ (right) channels, obtained by using the PACS-CS gauge configurations. $N_{\text {conf }}=422$ is used. 
energy obtained in the ${ }^{1} S_{0}\left({ }^{3} S_{1}\right)$ channel is $E=-1.9(1.3) \mathrm{MeV}(E=-1.3(1.2) \mathrm{MeV})$. The present results for the energies suggest that the spin dependence of the interaction in the low-energy region seems to be weak, although we need more statistics (and perhaps larger spatial volume) to make definite conclusion. The right panel of Figure 1 shows the effective central potential of $\Lambda p$ system obtained at the time slice $t-t_{0}=7$. The strong repulsive core is obtained in the ${ }^{1} S_{0}$ channel, while the repulsive core in the ${ }^{3} S_{1}$ channel is relatively weak. The left (right) inset shows the enlargement in the ${ }^{1} S_{0}\left({ }^{3} S_{1}\right)$ channel; A slight attractive well can be seen in the both spin channels.

\subsection{Quenched QCD}

We calculate the wave functions for two sets of the hopping parameters $\left(\kappa_{u d}, \kappa_{s}\right)=(0.1665,0.1643)$ and $(0.1670,0.1643)$. Table 1 also lists the hadron masses obtained from these quenched QCD calculations. The Figure 3 shows the wave function obtained with $\left(\kappa_{u d}, \kappa_{s}\right)=(0.1670,0.1643)$ at the time slice $t-t_{0}=7$, by using $N_{\text {conf }}=550$ gauge configurations. For both cases of calculations with $\left(\kappa_{u d}, \kappa_{s}\right)=(0.1670,0.1643)$ and $(0.1665,0.1643)$ in the ${ }^{1} S_{0}$ and in the ${ }^{3} S_{1}$ channels, the ground state saturations are achieved at the time slice $t-t_{0}=7$. The energies obtained by fitting the wave function in the asymptotic region $(10 \leq|\vec{r}| \leq 16)$ to the Green's function (4.2) in the ${ }^{1} S_{0}$ $\left({ }^{3} S_{1}\right)$ channel are $E=-0.37(12) \mathrm{MeV}(E=-0.47(11) \mathrm{MeV})$ for $\left(\kappa_{u d}, \kappa_{s}\right)=(0.1670,0.1643)$, and $E=-0.31(9) \mathrm{MeV}(E=-0.38(7) \mathrm{MeV})$ for $\left(\kappa_{u d}, \kappa_{s}\right)=(0.1665,0.1643)$, respectively. The lowest scattering energies in both ${ }^{1} S_{0}$ and ${ }^{3} S_{1}$ channels become lower as the $u, d$ quark mass decreases. We hardly find the clear conclusion whether the interaction in the ${ }^{1} S_{0}$ channel is more attractive than that in the ${ }^{3} S_{1}$ channel. Figure 4 shows the effective central potentials for $\Lambda p$ in the ${ }^{1} S_{0}\left({ }^{3} S_{1}\right)$ channel in the left (right) panel, obtained from the wave function. For both $\left({ }^{1} S_{0}\right.$ and $\left.{ }^{3} S_{1}\right)$ spin channels, the height of the repulsive core increases as the $u, d$ quark mass decreases.

In summary, we calculate the $\Lambda N$ potential from lattice QCD. The full lattice QCD calculation for the $\Lambda N$ system with larger volume and smaller lattice spacing at physical quark mass is highly desirable to clarify the spin dependence of the interaction.

\section{Acknowledgments}

The authors would like to thank PACS-CS Collaboration for allowing us to access their full

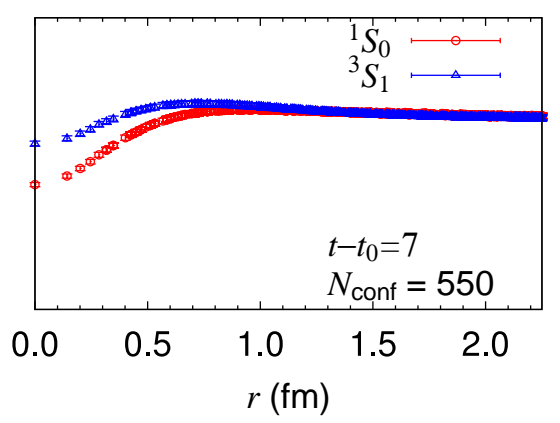

Figure 3: Left: The radial wave function of $\Lambda p$, in ${ }^{1} S_{0}$ (red circle) and ${ }^{3} S_{1}$ (blue triangle) channels, obtained by quenched QCD calculation with $\left(\kappa_{u d}, \kappa_{s}\right)=(0.1670,0.1643)$, at the time slice $t-t_{0}=7$. 

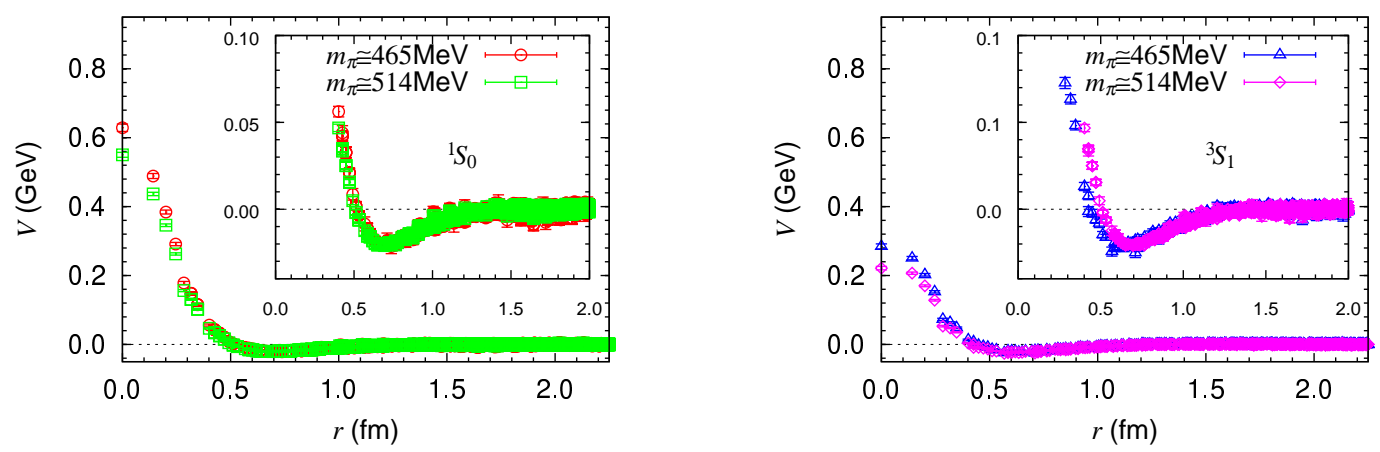

Figure 4: Left: The effective central potential for $\Lambda p$ in the ${ }^{1} S_{0}$ channel at $m_{\pi} \simeq 465 \mathrm{MeV}$ (red circle) and $m_{\pi} \simeq 514 \mathrm{MeV}$ (green box). Right: The effective central potential for $\Lambda p$ in the ${ }^{3} S_{1}$ channel at $m_{\pi} \simeq 465$ $\mathrm{MeV}$ (blue triangle) and $m_{\pi} \simeq 514 \mathrm{MeV}$ (magenta diamond). The inset shows its enlargement.

QCD gauge configurations, and Dr. T. Izubuchi for providing a sample FFT code. The full QCD calculations have been done by using PACS-CS computer under the "Interdisciplinary Computational Science Program" of Center for Computational Science, University of Tsukuba (No 08a-12). The Quenched QCD calculations have been done by using Blue Gene/L computer under the "Large scale simulation program" at KEK (No. 08-19). Part of numerical analysis has been done by using RIKEN super combined cluster system at RIKEN. This research was partly supported by the Ministry of Education, Science, Sports and Culture, Grant-in-Aid (Nos. 18540253, 19540261, 20028013, 20340047).

\section{References}

[1] Reviewed in O. Hashimoto and H. Tamura, Prog. Part. Nucl. Phys. 57, 564 (2006).

[2] H. Nemura, Y. Akaishi and Y. Suzuki, Phys. Rev. Lett. 89, 142504 (2002) [arXiv:nucl-th/0203013].

[3] N. Ishii, S. Aoki, T. Hatsuda, Phys. Rev. Lett. 99, 022001 (2007).

[4] H.Nemura, N.Ishii, S.Aoki and T.Hatsuda, arXiv:0806.1094 [nucl-th].

[5] S. Aoki, T. Hatsuda and N. Ishii, arXiv:0805.2462 [hep-ph].

[6] S. Aoki, J. Balog, T. Hatsuda, N. Ishii, K. Murano, H. Nemura and P. Weisz, in these proceedings.

[7] N. Ishii, S. Aoki and T. Hatsuda, in these proceedings.

[8] S. Muroya, A. Nakamura and J. Nagata, Nucl. Phys. Proc. Suppl. 129, 239 (2004).

[9] S. R. Beane et al. [NPLQCD Collab.], Nucl. Phys. A 794, 62 (2007).

[10] S. Aoki, et al. [PACS-CS Collab.], arXiv:0807.1661 [hep-lat].

[11] M. Lüscher, Nucl. Phys. B 354, 531 (1991).

[12] S. Aoki, et al. [CP-PACS Collab.], Phys. Rev. D71, 094504 (2005).

[13] R. Tamagaki and W. Watari, Prog. Theor. Phys. Suppl. 39, 23 (1967).

[14] J.J.de Swart, et al., Springer Tracts in Modern Physics 60, 138 (1971).

[15] CPS++ http://qcdoc.phys.columbia.edu/chuiwoo_index.html. 